\title{
THE COMPLEXITY OF ORBITS OF COMPUTABLY ENUMERABLE SETS
}

\author{
PETER A. CHOLAK, RODNEY DOWNEY, AND LEO A. HARRINGTON
}

\begin{abstract}
The goal of this paper is to announce there is a single orbit of the c.e. sets with inclusion, $\mathcal{E}$, such that the question of membership in this orbit is $\Sigma_{1}^{1}$-complete. This result and proof have a number of nice corollaries: the Scott rank of $\mathcal{E}$ is $\omega_{1}^{\mathrm{CK}}+1$; not all orbits are elementarily definable; there is no arithmetic description of all orbits of $\mathcal{E}$; for all finite $\alpha \geq 9$, there is a properly $\Delta_{\alpha}^{0}$ orbit (from the proof).
\end{abstract}

\section{INTRODUCTION}

In the classic paper [32], Post suggested that the study of the lattice of computably (recursively) enumerable (c.e.) sets was fundamental in computability theory. Post observed that, at the time, all known undecidability proofs worked by coding some "noncomputability", (coded by a certain kind of set) into the theory at hand thereby arguing that the relevant structures could emulate computation. He argued that for such proofs the central object was the notion of a computably enumerable set, being one which is effectively generated as the range of some effective process. The basic example is the set of consequences of a computably enumerable set of axioms for a formal system. Of course, the other key concept in computability was that of Turing [36] who introduced the notion of reducibility. Reducibilities are pre-orderings used to measure relative computational complexity.

The interplay of these two basic objects, (Turing) reducibility and effectively enumerated (c.e.) sets has a long and rich history. Clearly the computably enumerable sets under union and intersection form a lattice, denoted by $\mathcal{E}$. Their Turing degrees form an upper semilattice,

Date: August 31, 2021.

2000 Mathematics Subject Classification. Primary 03D25.

Research partially supported NSF Grants DMS-96-34565, 99-88716, 02-45167 (Cholak), Marsden Fund of New Zealand (Downey), DMS-96-22290 and DMS-9971137 (Harrington). Some of involved work was done partially while Cholak and Downey were visiting the Institute for Mathematical Sciences, National University of Singapore in 2005. These visits were supported by the Institute. 
denoted by $\mathcal{R}$. Ever since the ground breaking paper of Post, there has been a persistent intuition that structural properties of computably enumerable sets have reflections in their degrees, and conversely. In particular, definability in $\mathcal{E}$ should be linked with information content as measured by $\mathcal{R}$.

The simplest possible illustration of this is the fact that the complemented members of $\mathcal{E}$ are exactly the members of $\mathbf{0}$ the degree of the computable sets. An excellent and deep example is Martin's result that the Turing degrees of maximal sets are exactly the high computably enumerable Turing degrees ${ }^{1}$, (that is, their jumps are as complex as possible, $\mathbf{a}^{\prime}=\mathbf{0}^{\prime \prime}$ ), where a co-infinite set $A$ is a maximal c.e. set iff for all c.e. sets $B$, if $A \subseteq B$ then either $A={ }^{*} B$ or $B={ }^{*} \omega$, where $={ }^{*}$ denotes equivalence modulo the filter of finite sets. Since a set $A$ is finite iff every subset is complements in $\mathcal{E}$, it is natural to consider $\mathcal{E}^{*}, \mathcal{E}$ modulo the filter of finite sets. Thus $A$ is a maximal set iff it represents a co-atom in $\mathcal{E}^{*}$.

An original impetus for work on $\mathcal{E}^{*}$ was Post's Problem : Post observed that the coding inherent in all known undecidability proofs of the time were so faithful that all computably enumerable sets seemed to be either the $\mathbf{0}^{\prime}$ the degree of the halting problem, or $\mathbf{0}$ the degree of the computable sets. He asked if this was always the case. Post's problem is the following: Are there c.e. sets of intermediate Turing degree? That is there a computably enumerable degree $\mathbf{a}$ with $\mathbf{0}<\mathbf{a}<\mathbf{0}^{\prime}$. Post even suggested a programme to answer this question. Since complemented members of $\mathcal{E}$ are computable, but $\emptyset^{\prime}=\left\{x: \varphi_{x}(x) \downarrow\right\}$, the halting problem, had many infinite computably enumerable sets disjoint from it, perhaps a very "noncomplemented" member of $\mathcal{E}$ would turn out to be incomplete. Towards this goal, Post defined a computably enumerable set $A$ to be simple if $\omega-A$ was infinite and for all infinite computably enumerable $W, W \cap A \neq \emptyset$. Post constructed a simple set and then proved that a simple set $A$ is not of the same $m$-degree as $\mathbf{0}^{\prime}$. Post's programme was to come up with a thinness property of the complement of a c.e. set which would guarantee Turing incompleteness. Interpreted more liberally, we can generalize Post's programme and ask whether there is any definable property of a c.e. set in $\mathcal{E}^{*}$ which would guarantee Turing incompleteness.

While Post's problem was eventually solved by the development of the priority method independently by Friedberg [18] and Muchnik [31] out of the work of Kleene and Post [24], whether Post's Programme

\footnotetext{
${ }^{1}$ Indeed the reader should recall that, more generally, a set $A$ is $\operatorname{low}_{n}$ iff $A^{(n)}=\emptyset^{(n)}$ iff $\Delta_{n+1}^{0}=\Delta_{n+1}^{A}$, and $A$ is high $n$ iff $A^{(n)}=\emptyset^{(n+1)}$ iff $\Delta_{n+2}^{0}=\Delta_{n+1}^{A}$.
} 
could be realized successfully remained open for much longer. Myhill observed that if Post's original programme were to succeed then maximal sets, first constructed by Friedberg, should be Turing incomplete. On the other hand, rather than being Turing incomplete, Tennenbaum suggested that all maximal sets would be Turing complete. As we have have seen above, following earlier work of Tennenbaum, Sacks, and Yates, and others, Martin classified the degrees of maximal sets as precisely the high computably enumerable degrees. Martin's theorems demonstrate can be seen as a partial realization of Tennenbaum's intuition. That is, in spite of the fact that maximal sets may not necessarily be complete, they do have high information content in the sense that they have the same jump as the halting problem. That is, as far as the jump operator is concerned, they are indistinguishable from the halting problem. Martin had discovered the first invariant class in $\mathcal{R}$ in the sense that the high degrees are precisely those realized by the (definable class of) maximal sets.

Definability goes hand in hand with automorphisms of structures. Thus, a class $\mathcal{C}$ of computably enumerable degrees invariant if there is a collection $\mathcal{C}^{\prime}$ of computably enumerable sets closed under automorphisms of $\mathcal{E}$ such that $\mathcal{C}=\left\{\operatorname{deg}(A): A \in \mathcal{C}^{\prime}\right\}$. The following definition will be important for our subsequent discussion.

Definition 1.1. $A \approx \hat{A}$ iff there is a map, $\Phi$, from the c.e. sets to the c.e. sets preserving inclusion, $\subseteq$, (so $\Phi \in \operatorname{Aut}(\mathcal{E}))$ such that $\Phi(A)=\hat{A}$.

By Soare [34], $\mathcal{E}$ can be replaced with $\mathcal{E}^{*}$, since Soare showed that every automorphism of $\mathcal{E}^{*}$ is equivalent to one on $\mathcal{E}$ and conversely (as long we focus on infinite and co-infinite sets). Early work by Lachlan and others showed that both of the automorphism groups $\operatorname{Aut}(\mathcal{E})$ and $\operatorname{Aut}\left(\mathcal{E}^{*}\right)$ were large since each had $2^{\aleph_{0}}$ automorphisms. Martin [30] used a priority construction to show that a certain construction of Post (hypersimplicity) was not invariant under automorphisms of $\mathcal{E}$.

Post's original programme was to look at thinness properties of the lattice of the complement of a c.e. set $A$. This leads to a definition which will be important in the following.

Definition 1.2. $\mathcal{L}^{*}(A)$ is $\{W \cup A: W$ an c.e. set $\}$ under $\subseteq$ modulo the ideal of finite sets $(\mathcal{F})$. (The outside of a set.)

The final blow to Post's original programme was the marvelous paper of Soare [34], who showed that maximal sets form an orbit in $\operatorname{Aut}(\mathcal{E})$. In particular, no "extra" property together with maximality could guarantee incompleteness. Moreover, the paper Cholak, Downey and Stob [3], showed that no property of $\mathcal{L}^{*}(A)$ alone could guarantee Turing 
incompleteness for a computably enumerable set $A$. That is, Cholak, Downey and Stob proved that if for any computably enumerable set $A$ there is a set computably enumerable Turing complete $B$ with the same lattice of supersets as $A, \mathcal{L}^{*}(A) \cong \mathcal{L}^{*}(B)$.

Soare's [34] was highly influential. The methods introduced constructed $\Delta_{3}^{0}$ automorphisms of $\mathcal{E}^{*}$. Here we will classify automorphisms according to the complexity of a presentation of them. A presentation will be a function $f: \omega \rightarrow \omega$ such that that $W_{e} \mapsto W_{f(e)}$ induces the automorphism. If $e \mapsto f(e)$ a $\Delta_{3}^{0}$ function, we would call the automorphism $\Delta_{3}^{0}$, and $A \approx_{\Delta_{3}^{0}} \hat{A}$. While later papers presented Soare's automorphism machinery argument as a more thematic and flexible tree argument (beginning with Cholak [5] and [6] and Harrington and Soare [22]) most of the key underlying ideas for constructing automorphisms of $(\mathcal{E})$ are in Soare's original paper.

The principal tool used is called the (or, in view of recent work, an) Extension Lemma. Roughly speaking, constructing an automorphism works as follows. We wish to show maximal $A \approx \hat{A}$. We are given two versions of the universe $\omega$, called $\omega$ and $\hat{\omega}$ with $A \subset \omega$ and $\hat{A} \subset \hat{\omega}$, with enumerations of c.e. $\left\{W_{e}: e \in \omega\right\}$ subsets of $\omega$ and $\left\{V_{e}: e \in \omega\right\}$ subsets of $\hat{\omega}$. We must define some sort of mapping as follows

$$
\begin{array}{ll}
\omega & \mapsto \hat{\omega} \\
A & \mapsto \hat{A} \\
W_{e} & \mapsto \hat{W}_{f(e)} \\
\hat{V}_{g(e)} & \mapsto V_{e} .
\end{array}
$$

We must have the $\hat{W}_{f(e)}$ and $\hat{V}_{g(e)}$ so that we can argue that the mapping induces an automorphism of $\mathcal{E}^{*}$ by a back and forth argument. Here we are thinking of building the hatted sets $\hat{W}$ and $\hat{V}$. At the very least, intersections should be respected. That is, if $\bar{A} \cap W_{e}$ is infinite, then $\overline{\hat{A}} \cap \hat{W}_{f(e)}$ would need to be infinite. Similarly if $W_{e}-\hat{V}_{g(q)}$ is infinite the so too must be $\hat{W}_{f(e)}-V_{q}$, etc. Evidently, any possible diagram we can think of denoting intersections and difference would need to be respected. We represent these intersections and differences by states, which are strings measuring which $W_{e}$ 's and $\hat{V}_{k}$ 's on the $\omega$ side an element is in, and which $\hat{W}_{j}$ 's and $V_{q}$ 's a hatted element is in on the $\hat{\omega}$ side. We would write this basic requirement as

$$
\mathcal{R}_{\sigma}: \exists^{\infty} x \in \text { state } \sigma \text { iff } \exists^{\infty} \hat{x} \in \text { state } \hat{\sigma} \text {. }
$$

Soare's original idea is to begin on the $\mathcal{L}^{*}(A) \cong \mathcal{L}^{*}(\hat{A})$. He would make this an isomorphism and then extend this outside isomorphism 
to an automorphism by an isomorphism of the lattice of computably enumerable subsets of $A$ to those of $\hat{A}$.

Concentrating on the $\mathcal{L}^{*}(A) \cong \mathcal{L}^{*}(\hat{A})$ part, as we go along, elements appear to be in this region (that is, in $\bar{A}_{s}$ or $\overline{\hat{A}}_{s}$ ), and we build corresponding sets to match the states measuring intersections. This would seem not too hard in the case of a maximal set since for any sets $W$ either $W \cap \bar{A}$ is finite or $W$ almost contains $\bar{A}$. On the hatted side, all we would need to do is either have the corresponding $\hat{W}$ empty, or containing $\overline{\hat{A}}$, and similarly for the mappings from the hatted side back. The information as to which is correct is $\Sigma_{3}^{0}$ information and can be handled by a priority argument.

However, the heart of Soare's method is the following. As we go along enumerating hatted sets as elements stream into $\bar{A}_{s}$ many of these will be based on wrong information (such as the fact that at stage $s$ they might appear in the complement of $A$ yet might be in $A$ ), and will later enter $A$, and hence be in $W_{e} \searrow A$. They will enter $A_{t}$ and $\hat{A}_{u}$ in various entry states some caused by the $W_{e}$ and $V_{e}$ played by the opponent, and the hatted sets played by us. The key problem is how to handle these wrongly enumerated elements and be able to extend the $\mathcal{L}^{*}(A) \cong \mathcal{L}^{*}(\hat{A})$ correspondence to an automorphism. For the automorphism machinery to succeed, it is necessary to ensure that for all entry states $\sigma$ (and dually for $\hat{\sigma})$, if infinitely many elements enter $A$ in state $\sigma$, then there is some covering entry state $\hat{\tau}$ ( $\tau$, respectively). Here covering means that it is within our power to add elements into sets under our control to be able to match states.

Soare's Extension Lemma shows that this necessary condition is sufficient. Soare showed that we have not already killed that automorphism, meaning that the necessary condition is satisfied, then there is a strategy which enables us to extend the partial matching into a full automorphism. On the inside, that is the lattice of subsets of $A$ to those of $\hat{A}$ the map is $\Delta_{3}^{0}$, and in the case of maximal sets, Soare's original result shows that if $A$ and $\hat{A}$ are maximal, then $A \approx_{\Delta_{3}^{0}} \hat{A}$.

There is a lot of subsequent work on automorphisms and invariance in the lattice of c.e. sets. Almost all of it either uses Soare's original Extension Lemma as a black box, or modified it, to prove various results on the lattice of c.e. sets. Examples include the work of Maass [27], Maass and Stob [28], and Downey and Stob [16].

Early on, the methods seemed so powerful that anything seemed possible. Perhaps all sets were automorphic to complete sets, as suggested by Soare [35]. Certainly Harrington and Soare, and Cholak independently showed that all sets were automorphic to high sets. 
Hand in hand with this work constructing automorphisms was another line of investigation, where failures of the automorphism machinery could be exploited to provide definability results. A classic example of this is the work of Harrington and Soare [21] [23] who showed that the more general form of Post's Programme ${ }^{2}$ indeed has a positive solution.

Theorem 1.3 (Harrington and Soare [21]). There is a definable property $Q(A)$, such that, if a c.e. set $A$ satisfies $Q(A)$, then $A$ is Turing incomplete.

There were precursers to the Harrington-Soare result. Harrington used the idea of exploiting the failure of the machinery to get a definition of being a halting problem in the lattice of c.e. sets. Similarly Lerman and Soare [26] showed that there are low simple sets that are elementarily inequivalent, in that one has a property called d-simplicity and one has not, where d-simplicity is an elementary property implying certain facts about entry states. Another example of this can be found in Downey and Harrington [17] where the "no fat orbit" theorem is proven. The simplest form of the Downey-Harrington result below says that no c.e. set has an orbit hitting all nonzero degrees.

Theorem 1.4 (Downey and Harrington - No fat orbit). There is a property $S(A)$, a prompt low degree $\mathbf{d}_{\mathbf{1}}$, a prompt high $h_{2}$ degree $\mathbf{d}_{\mathbf{2}}$ greater than $\mathbf{d}_{\mathbf{1}}$, and tardy high ${ }_{2}$ degree e such that for all $E \leq_{T} \mathbf{e}, \neg S(E)$ and if $\mathbf{d}_{\mathbf{1}} \leq_{T} D \leq_{T} \mathbf{d}_{\mathbf{2}}$ then $S(D)$.

We remark that this "failure" methodology has yielded similar definability results in other structures such as the lattice of $\Pi_{1}^{0}$ classes, as witnessed by Weber [37] and [38], Cholak and Downey [11], and Downey and Montalbán [14]. Perhaps the best example of the methodology is the following proof of the definability of the double jump classes, the proof using "patterns" which are more or less direct reflections of blockages to the automorphism machinery.

Theorem 1.5 (Cholak and Harrington 02). Let $\mathcal{C}=\{\mathbf{a}: \mathbf{a}$ is the Turing degree of a $\Sigma_{3}$ set greater than $\left.\mathbf{0}^{\prime \prime}\right\}$. Let $\mathcal{D} \subseteq \mathcal{C}$ such that $\mathcal{D}$ is upward closed. Then there is an non-elementary $\left(\mathcal{L}_{\omega_{1}, \omega}\right) \mathcal{L}(A)$ property $\varphi_{\mathcal{D}}(A)$ such that $D^{\prime \prime} \in \mathcal{D}$ iff there is an $A$ where $A \equiv D$ and $\varphi_{\mathcal{D}}(A)$.

Corollary 1.6. If $\mathbf{a}^{\prime \prime}>\mathbf{b}^{\prime \prime}$ then there is a $A \in \mathbf{a}$ such that for all $B \in \mathbf{b}, A$ is not automorphic to $B$ (in fact, $\mathcal{L}^{*}(A) \neq \mathcal{L}^{*}(B)$ ).

\footnotetext{
${ }^{2}$ Another solution to Post's Programme was due to Marchenkov [29] who showed that a certain type of maximal set in a related quotient structure gave a solution.
} 
Related here is the following conjecture of Harrington.

Conjecture 1.7 (Harrington). For all $A$ and degrees $\mathbf{d}$ if $A^{\prime} \leq_{T} \mathbf{d}^{\prime}$ is there $\hat{A} \in \mathbf{d}$ such that $\mathcal{L}^{*}(A) \cong \mathcal{L}^{*}(\hat{A})$.

For more of these results one can see the paper [12].

\section{New Results}

The present work is motivated by basic questions about the automorphism group of $\mathcal{E}^{*}$. How complicated is it? If $A \approx \hat{A}$ is $A \approx \hat{A}$ witnessed by an arithmetical automorphism? How complicated is $\left\{W_{e}: W_{e} \approx A\right\}$ for a fixed $A$ ? The following conjecture was made by Ted Slaman and Hugh Woodin in 1989.

Conjecture 2.1 (Slaman and Woodin [33]). The set $\left.\left\{\langle i, j\rangle: W_{i} \approx W_{j}\right)\right\}$ is $\Sigma_{1}^{1}$-complete.

This conjecture was claimed to be true by the authors in the mid 1990s; but no proof appeared. One of the roles of this announcement and the full paper [9] is to correct that omission. The proof we will present is far simpler than all previous (and hence unpublishable) proofs. Indeed, much of the material reported in this paper due to Cholak and Harrington was developed towards making the proof of the conjecture accessible. The other important role of this communcation and the full paper is to prove a stronger result.

Theorem 2.2 (The Main Theorem). There is a c.e. set A such that the index set $\left\{i: W_{i} \approx A\right\}$ is $\Sigma_{1}^{1}$-complete.

As mentioned in the abstract this theorem does have a number of nice corollaries.

Corollary 2.3. Not all orbits are elementarily definable; there is no arithmetic description of all orbits of $\mathcal{E}$.

Corollary 2.4. The Scott rank of $\mathcal{E}$ is $\omega_{1}^{\mathrm{CK}}+1$.

Proof. Our definition that a structure has Scott rank $\omega_{1}^{\mathrm{CK}}+1$ is that there is an orbit such that membership in that orbit is $\Sigma_{1}^{1}$-complete. There are other equivalent definitions of a structure having Scott Rank $\omega_{1}^{\mathrm{CK}}+1$ and we refer the readers to Ash and Knight [1].

A consequence of the method of the proof (and some further effort to preserve quantifiers) is the following.

Theorem 2.5. For all finite $\alpha>8$ there is a properly $\Delta_{\alpha}^{0}$ orbit. 
Hitherto this paper [9] all known orbits were $\Delta_{3}^{0}$ with the single exception of the orbit of Cholak and Harrington [8] which constructed a pair of sets $\Delta_{5}^{0}$ automorphic but not $\Delta_{3}^{0}$.

Before we turn to the proof of Theorem 2.2, we will discuss the background to the Slaman-Woodin Conjecture. Certainly the set $\left.\left\{\langle i, j\rangle: W_{i} \approx W_{j}\right)\right\}$ is $\Sigma_{1}^{1}$. Why would we believe it to be $\Sigma_{1}^{1}$-complete? The following result is from the folklore ${ }^{3}$.

Theorem 2.6 (Folklore $\left.{ }^{4}\right)$. There is a computable listing, $\mathcal{B}_{i}$, of computable Boolean algebras such that the set $\left\{\langle i, j\rangle: \mathcal{B}_{i} \cong \mathcal{B}_{j}\right\}$ is $\Sigma_{1}^{1}$ complete.

Note that $\mathcal{L}^{*}(A)$ is a definable structure in $\mathcal{E}$ with a parameter for $A$. The following result says that the full complexity of the isomorphism problem for Boolean algebras of Theorem 2.6 is present in the supersets of a c.e. set.

Theorem 2.7 (Lachlan [25]). Effectively in $i$ there is a c.e. set $H_{i}$ such that $\mathcal{L}^{*}\left(H_{i}\right) \cong \mathcal{B}_{i}$.

Corollary 2.8. The set $\left\{\langle i, j\rangle: \mathcal{L}^{*}\left(H_{i}\right) \cong \mathcal{L}^{*}\left(H_{j}\right)\right\}$ is $\Sigma_{1}^{1}$-complete.

Slaman and Woodin's idea was to replace " $\mathcal{L}^{*}\left(H_{i}\right) \cong \mathcal{L}^{*}\left(H_{j}\right)$ " with " $H_{i} \approx H_{j}$ ". Unfortunately, this very attractive idea is doomed, as we now see.

Definition 2.9 (The sets disjoint from $A$ ).

$$
\mathcal{D}(A)=\left(\left\{B: \exists W\left(B \subseteq A \cup W \text { and } W \cap A={ }^{*} \emptyset\right)\right\}, \subseteq\right) .
$$

Let $\mathcal{E}_{\mathcal{D}(A)}$ be $\mathcal{E}$ modulo $\mathcal{D}(A)$. $A$ is $\mathcal{D}$-hhsimple iff $\mathcal{E}_{\mathcal{D}(A)}$ is a Boolean Algebra. $A$ is $\mathcal{D}$-maximal iff $\mathcal{E}_{\mathcal{D}(A)}$ is the trivial Boolean Algebra.

Lemma 2.10. If $A$ is simple then $\mathcal{E}_{\mathcal{D}(A)} \cong{ }_{\Delta_{3}^{0}} \mathcal{L}^{*}(A)$.

It is an old result of Lachlan [25] that $A$ is hhsimple iff $\mathcal{E}_{\mathcal{D}(A)}$ is a Boolean algebra. Except for the creative sets, until recently, all known orbits were orbits of $\mathcal{D}$-hhsimple sets. We direct the reader to Cholak

\footnotetext{
${ }^{3}$ We think it is well known that the isomorphism problem for Boolean Algebras and Trees are $\Sigma_{1}^{1}$-complete, at least in the form stated in Theorems 2.6 and 5.1. We have searched for a reference to a proof for these theorems without success. It seems very likely that these theorems were known to Kleene. There are a number of places where something very close to what we want appears; for example, see the example at the end of Section 5 of Goncharov et al. [19] and surely there are earlier examples (for example, White [39]). All of these constructions work by coding the Harrison ordering. In the full paper we give self-contained proofs of the folklore theorems we use.

${ }^{4}$ See Section 5 of the full paper [9] for more information and a proof.
} 
and Harrington [8] for a further discussion of this claim and for an orbit of $\mathcal{E}$ which does not contain any $\mathcal{D}$-hhsimple sets. The following are relevant theorems from Cholak and Harrington [8].

Theorem 2.11. If $A$ is $\mathcal{D}$-hhsimple and $A$ and $\hat{A}$ are in the same orbit then $\mathcal{E}_{\mathcal{D}(A)} \cong_{\Delta_{3}^{0}} \mathcal{E}_{\mathcal{D}(\hat{A})}$.

Theorem 2.12 (using Maass [27]). If $A$ is $\mathcal{D}$-hhsimple and simple (i.e., hhsimple) then $A \approx \hat{A}$ iff $\mathcal{L}^{*}(A) \cong{ }_{\Delta_{3}^{0}} \mathcal{L}^{*}(\hat{A})$.

Hence the Slaman-Woodin plan of attack fails. In fact even more is true.

Theorem 2.13. If $A$ and $\hat{A}$ are automorphic then $\mathcal{E}_{\mathcal{D}(A)}$ and $\mathcal{E}_{\mathcal{D}(\hat{A})}$ are $\Delta_{6}^{0}$-isomorphic.

Hence in order to prove Theorem 2.2 we must code everything into $\mathcal{D}(A)$. This is completely contrary to all approaches used to try to prove the Slaman-Woodin Conjecture over the years. We will point out two more theorems from Cholak and Harrington [8] to show how far the sets we use for the proof must be from simple sets, in order to prove Theorem 2.2.

Theorem 2.14. If $A$ is simple then $A \approx \hat{A}$ iff $A \approx_{\Delta_{6}^{0}} \hat{A}$.

Theorem 2.15. If $A$ and $\hat{A}$ are both promptly simple then $A \approx \hat{A}$ iff $A \approx_{\Delta_{3}^{0}} \hat{A}$.

\section{Future Work and the Degrees of the Constructed ORBITS}

While this work does answer many open questions about the orbits of c.e. sets, there are many questions left open. But perhaps these open questions are of a more degree-theoretic flavor. We will list three questions here.

Question 3.1 (Completeness). Which c.e. sets are automorphic to complete sets?

Of course, by Harrington and Soare [21], we know that not every c.e. set is automorphic to a complete set, and partial classifications of precisely which sets can be found in Downey and Stob [16] and Harrington and Soare [22, 20].

Question 3.2 (Cone Avoidance). Given an incomplete c.e. degree $\mathbf{d}$ and an incomplete c.e. set $A$, is there an $\hat{A}$ automorphic to $A$ such that $\mathrm{d} \not_{T} \hat{A}$ ? 
Question 3.3 (Can single jumps be coded into $\mathcal{E}$ ?). Let $J$ be C.E.A. in $\mathbf{0}^{\prime}$ but not of degree $\mathbf{0}^{\prime \prime}$. Is there a degree a such that $\mathbf{a}^{\prime} \equiv_{T} J$ and, for all $A \in \mathbf{a}$, there is an $\hat{A}$ with $A$ automorphic to $\hat{A}$ and $\hat{A}^{\prime}<_{T} \mathbf{a}^{\prime}$ or $\left.\hat{A}^{\prime}\right|_{T} \mathbf{a}^{\prime}$ ?

Question 3.4 (Can a single Turing degree be coded into $\mathcal{E}$ ?). Is there a degree $\mathbf{d}$ and an incomplete set $A$ such that, for all $\hat{A}$ automorphic to $A, \mathbf{d} \leq \hat{A}$ ? $A \in \mathbf{d}$ ?

In a technical sense, these may not have a "reasonable" answer. Thus the following seems a reasonable question.

Question 3.5. Are these arithmetical questions?

In this paper we do not have the space to discuss the import of these questions. Furthermore, it not clear how this current work impacts possible approaches to these questions. At this point we will just direct the reader to slides of a presentation of Cholak [4]; perhaps a paper reflecting on these issues will appear later.

One of the issues that will impact all of these questions are which degrees can be realized in the orbits that we construct in Theorem 2.2 and 2.5. A set is hemimaximal iff it is the nontrivial split of a maximal set. A degree is hemimaximal iff it contains a hemimaximal set. Downey and Stob [16] proved that the hemimaximal sets form an orbit, and in some sense, this orbit is very large degree-theoretically. While it is known by Downey and Harrington [17] that there is no orbit containing sets of all nonzero degrees, the orbit of hemimaximal sets contain representatives of all jump classes (Downey and Stob [15]).

We are able to also show that we can construct our orbits to contain at least a fixed hemimaximal degree (possibly along others) or contain all hemimaximal degrees (again possibly along others). However, what is open is if every such orbit must contain a representative of every hemimaximal degree or only hemimaximal degrees. For the proofs of these claims, we direct the reader to Section 5.3.

\section{Past Work and Other Connections}

The paper [9] is a fourth paper in a series of loosely connected papers, the previous three being by Harrington and Cholak [13], [7], and [8]. We have seen above that results from [8] determine the direction one must take to prove Theorem 2.2. The above results from [8] depend heavily on the main result in [7] whose proof depends on special $\mathcal{L}$ patterns and several theorems about them which can be found in [13]. It is not necessary to understand any of the above-mentioned theorems from any of these papers to understand the proof of Theorem 2.2. 
But the proof of Theorem 2.2 does depend on Theorems 2.16, 2.17, and 5.10 of Cholak and Harrington [8]; see Section 5. The proof of Theorem 2.5 also needs Theorem 6.3 of Cholak and Harrington [8]. The first two theorems are straightforward but the third and fourth require work. The third is another modified "Extension Theorem." The fourth is what we might call a "Restriction Theorem"; it restricts the possibilities for automorphisms.

Fortunately, we are able to use these four theorems from Cholak and Harrington [8] as black boxes. These four theorems provide a clean interface between the two papers. If one wants to understand the proofs of these four theorems one must go to Cholak and Harrington [8]; otherwise, the paper [9] is completely independent from its three predecessors. In the next section we will explore the statements of Theorems 5.10 and 6.3 of Cholak and Harrington [8] in more detail.

4.1. An Algebraic Framework. Crucial to the Theorem 2.2 is the following theorem of the second two authors which demonstrates that $\Delta_{3}^{0}$ Extension Lemmas are central to our understanding of the automorphism group of $\mathcal{E}^{*}$.

Theorem 4.1 (Theorem 6.3 of Cholak and Harrington [8]). Assume $D$ and $\hat{D}$ are automorphic via $\Psi$. Then $D$ and $\hat{D}$ are automorphic via $\Theta$ where $\Theta\left\lceil\mathcal{E}(D)\right.$ is $\Delta_{3}^{0}$.

Theorem 4.1 says that inside any automorphism can be thought of as $\Delta_{3}^{0}$. The proof of this result and others we will need relies heavily on the framework of the second two authors who have recast the idea of an Extension Lemma algebraically so that the dynamic notions of entry states and matching are replaced by extendible Boolean algebras and supports. In particular, these proofs relies on Theorem 4.5. We will briefly discuss these methods of Cholak and Harrington.

Fix a c.e. set $A$. Then the structure $\mathcal{S}(A)=\{B: \exists C(C \sqcup B=A)\}$, the Boolean algebra of (c.e.) splits of $A$. Let $\mathcal{R}(A)=\{R: R \subseteq A$ and $R$ computable\}, with $\mathcal{S}_{R}(A)$ the quotient of $\mathcal{S}(A)$ by $\mathcal{R}(A)$, and $=^{R}, \subseteq^{R}$ the corresponding quotient relations. It is proven in Cholak and Harrington [8] that $\mathcal{S}_{R}(A)$ is always a $\Sigma_{3}^{0}$ Boolean algebra. Ones that have representations of low complexity are especially important to us. A uniformly computable listing $\mathcal{S}=\left\{S_{i}: i \in \omega\right\}$ of splits of $A$ is called an effective listing of splits of $A$ iff there exists another uniformly computable listing of splits of $A,\left\{\hat{S}_{i}: i \in \omega\right\}$, with $S_{i} \sqcup \hat{S}_{i}=A$ for all $i$. The idea here is based around the fact that those elements $x$ in $W_{e}$ and then later enter $A$ give rise to effective listings of splits. 
Definition 4.2 (Cholak and Harrington [8]). A $\Sigma_{3}^{0}$ subalgebra $\mathcal{B}$ of $\mathcal{S}_{R}(A)$ is called extendible iff there exists a representation $\mathcal{S}$ and $B$ of $\mathcal{B}$ such that $\mathcal{S}$ is an effective listing of splits of $A$ and $B$ is a $\Delta_{3}^{0}$ set.

Again following Cholak and Harrington [8], we consider a partial map $\Theta$ between splits of $A$ and splits of $\hat{A}$ (for general sets $A$ and $\hat{A})$ to be an isomorphism between a substructure $\mathcal{B}$ of $\mathcal{S}_{R}(A)$ and a substructure $\hat{B}$ of $\mathcal{S}_{R}(\hat{A})$, iff $\Theta$ preserves $\subseteq^{R}$, for each equivalence class $S_{R}$ of $\mathcal{B}$, if $S \in S_{R}$ then $\Theta(S)$ exists, and for each equivalence class $\hat{\mathcal{S}}_{R}$ of $\hat{B}, \Theta^{-1}(\hat{S})$ exists for all $\hat{S} \in \hat{\mathcal{S}}_{R}$. Then two extendible algebras $\mathcal{B}$ and $\hat{B}$ are extendibly isomorphic via $\Theta$ iff

(1) There is an effective listing $\mathcal{S}$ and $B$ witnessing that $\mathcal{B}$ is an extendible algebra.

(2) There is an effective listing $\hat{S}$ and $\hat{B}$ witnessing that $\hat{\mathcal{B}}$ is an extendible alga.

(3) For all $i \in B$, there is a $j \in \hat{B}$, with $\Theta\left(S_{i}\right)=\hat{S}_{j}$, and

(4) For each $j \in \hat{B}$, there is an $i \in B$ with $\Theta\left(S_{i}\right)=\hat{S}_{j}$.

(5) The partial map $\Theta^{\prime}$ indiced by $\Theta$ describes an isomorphism between $\mathcal{B}$ and $\hat{\mathcal{B}}$, as above.

The first algebraic version of the Extension Lemma is the following.

Theorem 4.3 (Cholak and Harrington Cholak and Harrington [8]). Let $\mathcal{B} \subseteq \mathcal{S}_{R}(A)$ and $\hat{\mathcal{B}}_{R} \subseteq \mathcal{S}_{R}(\hat{A})$ be two extendible Boolean algebras, which are $\Delta_{3}^{0}$ extendibly isomorphic via $\Theta$. Then there is a $\Phi$ which is a $\Delta_{3}^{0}$ isomorphism between $\mathcal{E}^{*}(A)$ and $\mathcal{E}^{*}(\hat{A})$, such that for all $i \in B$, $\Phi\left(S_{i}\right)={ }_{R} \Theta\left(S_{i}\right)$, and for all $i \in \hat{B}, \Phi^{1}\left(\hat{S}_{i}\right)={ }_{R} \Theta^{-1}\left(\hat{S}_{i}\right)$.

The key idea here is that it is possible to extend the extendible isomorphism between $\mathcal{B}$ and $\hat{\mathcal{B}}$ to an isomorphism between $\mathcal{E}^{*}(A)$ and $\mathcal{E}^{*}(\hat{A})$. Of course, nothing comes without price, and the proof of this (and similar) results, rely on dynamic extension lemmas, of one type or another. In the paper Cholak and Harrington [8], Theorem 4.3 is proven using a modification of Cholak's Translation Theorem, Cholak [2]

Whilst it is not directly pertinent to the present paper, we point out how Cholak and Harrington applied theorems like Theorem 4.3 using the idea of supports. This notion is related to the relationship between $\mathcal{L}^{*}(A)$ and $\mathcal{B}$, in some sense focusing on the relationship between the outside and the inside. We say a c.e. set $S$ supports $X$ if $S \subseteq X$ and $(X-A) \sqcup S$ is c.e.. For example, $W_{e} \searrow A$, the elements of $W_{e}$ which begin outside of $A_{s}$ and then later enter $A$ support $W_{e}$. More generally, an extendible algebra $\mathcal{B}$ supports a substructure $\mathcal{L}$ of $\mathcal{L}^{*}(A)$ 
(a subcollection of $\left\{W_{e} \cup A, \cup\right\}$ modulo finite sets), if for all $W \in \mathcal{L}$ there is an $i \in B$ with $S_{i}$ supporting $W$.

Definition 4.4 (Cholak and Harrington Cholak and Harrington [8]). Assume that $\mathcal{L}^{*}(A)$ and $\mathcal{L}^{*}(\hat{A})$ are isomorphic via $\Psi, \mathcal{B}$ and $\hat{\mathcal{B}}$ are extendible algebras isomorphic via $\Theta, \mathcal{B}$ supports $\mathcal{L}$ and $\hat{\mathcal{B}}$ supports $\hat{\mathcal{L}}$. Then we say that $\Psi$ and $\Theta$ preserve the supports if for $W \in \mathcal{L}$, there is an $i \in B$ such that $S_{i}$ supports $W$ and $(\Psi(W \cup A)-\hat{A}) \sqcup \Theta\left(S_{i}\right)$ is c.e., and for all $\hat{W} \in \hat{\mathcal{L}}$, there is an $i \in \hat{\mathcal{B}}$ with $\hat{S}_{i}$ supporting $\hat{W}$ and $\left.\Psi^{-1}(\hat{W} \cup \hat{A})-A\right) \sqcup \Theta^{-1}\left(\hat{S}_{i}\right)$ is c.e..

Then one algebraic version of the Extension Lemma is the following.

Theorem 4.5 (Cholak and Harrington [8]). Assume that $\mathcal{L}^{*}(A)$ and $\mathcal{L}^{*}(\hat{A})$ are isomorphic via $\Psi, \mathcal{B}$ and $\hat{\mathcal{B}}$ are extendible algebras isomorphic via $\Theta, \mathcal{B}$ supports $\mathcal{L}^{*}(A)$ and $\hat{\mathcal{B}}$ supports $\mathcal{L}^{*}(\hat{A})$, with $\Psi$ and $\Theta$ preserving supports.

Then there is an automorphism $\Lambda$ of $\mathcal{E}^{*}$ with $\Lambda(A)=\hat{A}, \Lambda \uparrow \mathcal{L}^{*}(A)=\Psi$, and such that $\Lambda \uparrow \mathcal{E}^{*}(A)$ is $\Delta_{3}^{0}$.

For reasons which become clear later, one final result from Cholak and Harrington's paper we will need concerns extendible algebras of computable sets. An extendible algebra $\mathcal{B}$ of $\mathcal{S}_{R}(\omega)$ is called a extendible algebra of computable sets, as the splits of $\omega$ are computable sets.

Theorem 4.6 (Theorem 5.10 of Cholak and Harrington [8]). Let $\mathcal{B}$ be an extendible algebra of computable sets and similarly for $\hat{\mathcal{B}}$. Assume the two are extendibly isomorphic via $\Pi$. Then there is a $\Phi$ such that $\Phi$ is a $\Delta_{3}^{0}$ isomorphism between $\mathcal{E}^{*}(A)$ and $\mathcal{E}^{*}(\hat{A}), \Phi$ maps computable subsets to computable subsets, and, for all $R \in \mathcal{B},(\Pi(R)-\hat{A}) \sqcup \Phi(R \cap A)$ is computable (and dually).

4.2. Some Algebraic Orbits. In [8], Cholak and Harrington use Theorem 4.5 to give algebraic proofs of many known theorems from the literature such as the maximal and hemimaximal results. (Additionally they use the algebraic methods for new results such as new orbits.) We remark that the algebraic view does go back to Herrmann's proof that a certain class of sets (now called Hermann sets) were automorphic. This result was proven using a hitherto unobserved algebraic consequence of the original Soare paper about the preservation of computable sets under Soare's construction. Given that is not well understood and is a critical proof of the Theorem 2.2, we would like to explore these algebraic proofs with some more detail. 
Definition 4.7. $\mathcal{C}(A)$ is the set of $W_{e}$ such that either $\bar{A} \subseteq W_{e}$ or $W_{e} \subseteq^{*} A$.

Theorem 4.8 (Soare's Automorphism Theorem [34]). Let $A$ and $\hat{A}$ be two noncomputable computably enumerable sets.

(1) Then there is a $\Delta_{3}^{0}$ isomorphism $\Lambda$ between $\mathcal{E}(A) \cup \mathcal{C}(A)$ and $\mathcal{E}(\hat{A}) \cup \mathcal{C}(\hat{A})$. Furthermore a $\Delta_{3}^{0}$-index for $\Lambda$ can be found uniformly from indexes for $A$ and $\hat{A}$.

(2) In addition, $\Lambda$ preserves the computable subsets of $A$.

Soare [34] explicitly stated Theorem 4.8.1. Theorem 4.8.2 was observed, in unpublished work, by Herrmann. Assume that $R$ is a computable subset of $A$. Herrmann's observation was that $\bar{R} \in \mathcal{C}(A)$ and hence $\Lambda(R) \sqcup \Lambda(\bar{R})={ }^{*} \hat{\omega}$ and therefore $\Lambda$ maps $R$ to a computable subset of $\hat{A}$. This observation of Herrmann was never published and is one of the key facts he used in showing that the Herrmann sets form an orbit; see Cholak et al. [10].

Theorem 4.9 (Soare [34]). The maximal sets form an orbit.

Proof. Assume that $A$ and $\hat{A}$ are maximal. Then $\mathcal{C}(A)=\mathcal{E}$. If $W \subseteq A$ then let $\Psi(W)=\Lambda(W)$. If $W \cup A={ }^{*} \omega$ there is a computable set $R_{W}$ such that $R_{w} \subseteq^{*} A$ and $\overline{R_{W}} \subseteq \subseteq^{*} A$ and then let $\Psi(W)=\Lambda\left(W \cup R_{W}\right) \sqcup \overline{\Lambda\left(R_{W}\right)}$. It is not difficult to show $\Psi$ is an automorphism.

Recall that set is hemimaximal iff it is the nontrivial split of a maximal set.

Theorem 4.10 (Downey and Stob [16]). The hemimaximal sets form an orbit.

Proof. Assume $A_{1} \sqcup A_{2}=A$ where the $A_{i}$ s are not computable and $A$ is maximal. Dually for $\hat{A}$. Assume that $\Theta_{i}$ is an isomorphism from $\mathcal{E}^{*}\left(A_{i}\right)$ to $\mathcal{E}^{*}\left(\hat{A}_{i}\right)$ that preserves the computable subsets (from Theorem 4.8).

As with the maximal sets, it is enough to define an isomorphism $\Lambda$ between $\mathcal{E}^{*}(A)$ and $\mathcal{E}^{*}(\hat{A})$ preserving the computable subsets. If $X \subseteq^{*} A$ then let $\Lambda(X)=\Theta_{1}\left(X \cap A_{1}\right) \sqcup \Theta_{2}\left(X \cap A_{2}\right)$. Let $R \in \mathcal{R}(A)$. Then $R \cap A_{i}$ is computable. So $\Theta_{i}\left(R \cap A_{i}\right)$ is computable. Hence $\Theta_{1}\left(R \cap A_{1}\right) \sqcup \Theta_{2}\left(R \cap A_{2}\right)$ is computable. The complexity of the resulting automorphism is $\Delta_{3}^{0}$.

Definition 4.11. We say that a c.e. set $H$ is strongly $r$-separable if, for all c.e. sets $W$ disjoint from $H$, there is a computable set $R$ such that $W \subset R, H \subset \bar{R}$, and $R-W$ is infinite. We say that a set $H$ is Herrmann if it is both $\mathcal{D}$-maximal and strongly $r$-separable. 
Theorem 4.12 (Herrmann, see Cholak et al. [10]). The Herrmann sets form an orbit (under $\Delta_{3}^{0}$ automorphisms).

Proof. Let $H$ be a Herrmann set. Since $H$ is $\mathcal{D}$-maximal for all $W$ there is a $W_{W}$ such that either $W \subseteq \subseteq^{*} H \sqcup W_{W}$ or $\bar{W} \subseteq^{*} H \sqcup W_{W}$. Furthermore, since $H$ is Herrmann, for all $W$, there is a computable $R_{W}$ such that either $W \subseteq^{*} H \sqcup R_{W}$ or $\bar{W} \subseteq^{*} H \sqcup R_{W}$. Note that finding $R_{W}$ and determining which case holds can be done using an oracle computable in $\mathbf{0}^{\prime \prime}$.

Assume $\bar{W} \subseteq \subseteq^{*} H \sqcup R_{W}$. Then $W \cup H \sqcup R_{W}=^{*} \omega$. Therefore $\left(W \cap \overline{R_{W}}\right) \cup H={ }^{*} \bar{R}_{W}$. Recall that $X \backslash Y=\left\{x \mid \exists s\left(x \in X_{s}-Y_{s}\right)\right\}$. Hence $\left(\left(W \cap \bar{R}_{W}\right) \backslash H\right) \sqcup\left(H \backslash\left(W \cap \bar{R}_{W}\right)\right)={ }^{*} \bar{R}_{W}$. Thus there is a computable subset $R_{H, W}=H \backslash\left(W \cap \bar{R}_{W}\right)$ of $H$ such that

$$
W={ }^{*}\left(W \cap R_{H, W}\right) \sqcup\left(\bar{R}_{H, W} \cap \bar{R}_{W}\right) \sqcup\left(W \cap R_{W}\right) .
$$

Again note that find $R_{H, W}$ can be using an oracle computable in $\mathbf{0}^{\prime \prime}$.

Now using $0^{\prime \prime}$ find a pairwise disjoint collection of $R_{i}$ such that $R_{i} \cap H=\emptyset$ and, for all $e, R_{W_{e}} \subseteq \bigsqcup_{i \leq e} R_{e}$. Since $H$ is Herrmann is possible to find such a collection. Do the same for $\hat{H}$.

Use Theorem 4.8 get $\Lambda$ mapping $\mathcal{E}(H)$ to $\mathcal{E}(\hat{H})$. Let $p_{i}$ be a computable one-to-one onto map from $R_{i}$ to $\hat{R}_{i}$ and, for $W \subseteq{ }^{*} R_{i}$, let $\Lambda_{i}(W)=p_{i}(W)$. All of these maps take computable subsets to computable subsets.

Now we will work on defining our automorphism $\Phi$. First assume $W \subseteq^{*} H \sqcup \sqcup R_{i}$. Let $\Phi(W)=\Lambda(H \cap W) \sqcup \sqcup \Lambda_{i}\left(R_{i} \cap W\right)$. It is not hard to show for $\Phi$, as defined so far, is order-preserving, for all $W, \Phi(W)$ is an r.e. set, and if $W$ is computable so is $\Phi(W)$.

Now consider the case when $W \nsubseteq^{*} H \sqcup \sqcup R_{i}$. In that case, Equation 4.12.1 holds, and we can use that to define $\Phi(W)$ in terms of subsets of $H \sqcup \sqcup R_{i}$ :

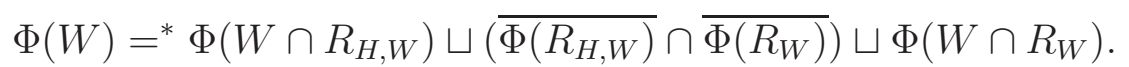

As defined $\Phi(W)$ is an r.e. set. It is not difficult show $\Phi$ is order preserving and hence well-defined. Thus $\Phi$ is an automorphism taking $H$ to $\hat{H}$.

For more on preserving the computable sets and an algebraic proof of Theorem 4.8 we refer the reader to Cholak and Harrington [8]. 


\section{A Sketch of the Proof of Theorem 2.2}

The proof of Theorem 2.2 is quite complex and involves several ingredients. The proof will be easiest to understand if we introduce each of the relevant ingredients in context.

The following theorem will prove be to useful.

Theorem 5.1 (Folklore $\left.{ }^{5}\right)$. There is a computable listing $T_{i}$ of computable infinite branching trees and a computable infinite branching tree $T_{\Sigma_{1}^{1}}$ such that the set $\left\{i: T_{\Sigma_{1}^{1}} \cong T_{i}\right\}$ is $\Sigma_{1}^{1}$-complete.

The idea for the proof of Theorem 2.2 is to code each of the above $T_{i}$ s into the orbit of $A_{T_{i}}$. Informally let $\mathcal{T}\left(A_{T}\right)$ denote this encoding; $\mathcal{T}\left(A_{T}\right)$ will not be defined in this announcement. But we will discuss in some details some of the ingredients and resulting complexity. The game plan is as follows:

(1) Coding: For each $T$ build an $A_{T}$ such that $T \cong \mathcal{T}\left(A_{T}\right)$ via an isomorphism $\Lambda \leq_{T} \mathbf{0}^{(\mathbf{2})}$.

(2) Coding is preserved under automorphic images: If $\hat{A} \approx A_{T}$ via an automorphism $\Phi$ then $\mathcal{T}(\hat{A})$ exists and $\mathcal{T}(\hat{A}) \cong T$ via an isomorphism $\Lambda_{\Phi}$, where $\Lambda_{\Phi} \leq_{T} \Phi \oplus \mathbf{0}^{(\mathbf{2})}$.

(3) Sets coding isomorphic trees belong to the same orbit: If $T \cong \hat{T}$ via isomorphism $\Lambda$ then $A_{T} \cong A_{\hat{T}}$ via an automorphism $\Phi_{\Lambda}$ where $\Phi_{\Lambda} \leq_{T} \Lambda \oplus \mathbf{0}^{(\mathbf{2})}$.

Thus $A_{T_{\Sigma_{1}^{1}}}$ and $A_{T_{i}}$ are in the same orbit iff $T_{\Sigma_{1}^{1}}$ and $T_{i}$ are isomorphic. Since the latter question is $\Sigma_{1}^{1}$-complete so is the former question.

We will build a pairwise disjoint collection of $D_{\chi}$ s to code the tree T. $A_{T}=D_{\lambda}$ will code the empty node in $T$. The basic module to construct an $D$ involves the construction of a computable set $R$ and a subset $M$ of $R$. There will be infinitely many pairwise disjoint $R$ s. Inside $R$ s the $D$ s will be Friedberg splits of $M$. It is well understood how to split an r.e. set $M$ into Friedberg splits.

Depending on the construction either $M={ }^{*} R$ or $M$ is maximal inside $R$; i.e. $M \cup \bar{R}$ is maximal. If $M$ is maximal inside $R$ then $D$ is hemimaximal inside $R$ and we say $D$ lives in $R$. If $M={ }^{*} R$ then, inside $R, D$ is a computable set and $D$ does not live in $R$. Note that $D$ living in $R$ is a definable property as is $D$ is computable in $R$.

The big issue of the construction will be to decide when $D$ lives in $R$ and when not. It is well understood how to construct an maximal set inside $R$. Furthermore it also well understood how use a dumping argument to alter the maximal set construction to force $M={ }^{*} R$.

\footnotetext{
${ }^{5}$ See Section 5 of the full paper for more information and a proof.
} 
We will use a dumping construction to alter the construction of the desired $M$. The decision whether $D$ lives in $R$ will be handled by a tree argument. Here we will not discuss the tree argument but discuss issues that go into deciding whether $D$ lives in $R$.

Each node $\chi$ of $T$ will have infinitely many pairwise disjoint $R_{\chi, i}$ associated with $\chi$. For these $R_{\chi, i}$ the corresponding set is $M_{\chi, i}$. $D_{\chi}$ will always be a Friedberg split of $M_{\chi, i}$. If $\chi^{+}$is a successor of $\chi$ in $T$ then, for almost all $R_{\chi, i}, D_{\chi^{+}}$be a Friedberg split of $M_{\chi, i}$. The collection of the all $R_{\chi, i}$ will be pairwise disjoint. When constructed in this fashion the $D_{\chi}$ code $T$ and hence we have part 1 of the game plan under control.

We have to work on part 2 and 3 of the game plan. For part 3 we are going to set things up so that if we know where the $D_{\chi}$ and $R_{\chi, i}$ go then we will be able to construct the desired automorphism.

Lets look at the hatted side of the construction briefly to work on Part 2. We are told $A_{T}$ goes to $\hat{A}$. We can gather together a collection of pairwise disjoint computable sets $\hat{R}_{\lambda, i}$ such that either $\hat{A}$ lives in $\hat{R}_{\lambda, i}$ or $\hat{A}$ is computable inside $\hat{R}_{\lambda, i}$. We can assume that this list is maximal; i.e. if $\hat{A}$ lives in $\hat{R}$ or is computable in $\hat{R}$, then $\hat{R} \subseteq \bigsqcup \hat{R}_{\lambda, i}$. There may many such lists. But we can show modulo a computable set each $R_{\lambda, i}$ must be sent to some $\hat{R}_{\lambda, j}$.

Now we are in position to pick out the successors of $\hat{A}$ on the hatted side. They are the sets $\hat{D}_{\lambda^{+}}$which live into almost all of $\hat{R}_{\lambda, i}$ that $\hat{A}$ lives in. More or less these sets must be the automorphic images of the $D_{\lambda^{+}, i}$ s. We better ensure that these sets behave like a good successor. The only way to do is to control how the $D_{\lambda^{+}, i}$ s behave.

Any set $D$ which looks like a $D_{\chi}$ must be split of one of the $D_{\chi}$ s. If, for infinitely many $\chi$ and $i, D$ lives in $R_{\chi, i}$ then there must be exactly one $\chi$ such that, for all $i, D$ lives in $R_{\chi, i}$ iff $D_{\chi}$ lives in $R_{\chi, i}$ and for almost all $i, D$ lives in $R_{\chi^{-}, i}$ iff $D_{\chi^{-}}$lives in $R_{\chi^{-}, i}$. In this case $D$ will be a Friedberg split of $D_{\chi}$.

If we can get the $D$ to behave properly (as discussed above) than their automorphic images must also behave properly. So the $\hat{D}_{\lambda^{+}}$which we have found above in fact code the successors of $\lambda$ in $\hat{T}$. Once we have this we can find their successors.

For each $\lambda^{+}=\hat{\chi}$ we can find a collection of pairwise disjoint computable sets $\hat{R}_{\hat{\chi}, i}$ such that either $\hat{D}_{\hat{\chi}}$ lives in $\hat{R}_{\hat{\chi}, i}$ or $\hat{D}_{\hat{\chi}}$ is computable inside $\hat{R}_{\hat{\chi}, i}$. We can assume that this list is maximal; i.e. if $\hat{D}_{\hat{\chi}}$ lives in $\hat{R}$ or is computable in $\hat{R}$, then $\hat{R} \subseteq \bigsqcup \hat{R}_{\hat{\chi}, i} \sqcup \bigsqcup \hat{R}_{\lambda, i}$. There may many such lists. Given that $\hat{D}_{\hat{\chi}}$ does code a successor of $\hat{A}$, there is some 
node $\chi$ of length 1 such that modulo a computable set each $R_{\chi, i}$ must be sent to some $\hat{R}_{\hat{\chi}, j}$. Now we can bootstrap our way to find successor of $\hat{D}_{\hat{\chi}}$ and so on. Hence part 2 is now under control.

So, for each possible $D$, we will make sure that if either $D$ is hemimaximal inside finite many $R_{\chi, i}$ or $D$ behaviors as above; i.e. $D$ will be a Friedberg split of some $D_{\chi}$. To do this we will use a coherence/state argument not unlike the argument used to constructed maximal sets. The state of $R_{\chi, i}$ will be those $D$ which $D$ is hemimaximal in $R_{\chi, i}$. Determining the state of a set is $\Sigma_{3}^{0}$ rather than $\Sigma_{1}^{0}$. Hence this is another reason we must do this whole construction on a tree. If $R_{\chi, i}$ is in an low $e$-state then we must dump it. We dump $R_{\chi, i}$ by making $M_{\chi, i}={ }^{*} R_{\chi, i}$ as discussed above.

As for the collection of all $R_{\chi, i}$ we want them to have the property that for all $W$, either $W$ is a split of the $D_{\chi}$ s or there is a finite set $F$ of $\chi$ and $i$ such that either $W \subseteq^{*} \bigsqcup_{(\chi, i) \in F} R_{\chi, i}$ or $\bar{W} \subseteq^{*} \bigsqcup_{(\chi, i) \in F} R_{\chi, i}$. This cannot be achieved via an effective construction but is achievable on a tree. This has the side effect that there will be more sets $R_{\chi, i}$ where $M_{\chi, i}={ }^{*} R_{\chi, i}$.

We will also construct that $R_{\chi, i}$ such that for all $\chi$, the $R_{\chi^{-}, i}$ and $R_{\chi, i}$ form an extendible algebra of computable sets, $\mathcal{B}_{\chi}$. We wish to use Theorem 4.6. For each $\chi$, the map $R_{\chi, i}$ to $\hat{R}_{\hat{\chi}, i}$ is an extendible isomorphism. We want to claim that we can use these pieces and an isomorphism between $T$ and $\hat{T}$ to construct an automorphism not unlike what we did in Section 4.2 and, in particular, with the proof of Theorem 4.12. With one caveat this is the case.

The caveat is that if $\chi$ and $\hat{\chi}$ have the same length we want to ensure that $D_{\chi}$ lives in $R_{\chi, i}$ iff $\hat{D}_{\hat{\chi}}$ lives in $\hat{R}_{\hat{\chi}, i}$. This requires two more additions to the above description. First we must construct all $A_{T} \mathrm{~s}$ using the same tree construction. Second the coherence/state argument above must be extended so that we the above homogeneous is preserved. So if $M_{\chi, i}={ }^{*} R_{\chi, i}$ then for all $\hat{\chi}$ of the same length as $\chi$, $M_{\hat{\chi}, i}={ }^{*} R_{\hat{\chi}, i}$.

5.1. Invariants. It might appear that $\mathcal{T}(A)$ is an invariant which determines the orbit of $A$. But there is no reason to believe for an arbitrary $A$ that $\mathcal{T}(A)$ is well defined. The following theorem shows that $\mathcal{T}(\hat{A})$ is an invariant as far as the orbits of the $A_{T} \mathrm{~s}$ are concerned. In the full paper, we prove a more technical version of the following theorem.

Theorem 5.2. If $\hat{A}$ and $A_{T}$ are automorphic via $\Psi$ and $T \cong \mathcal{T}(\hat{A})$ via $\Lambda$ then $A_{T} \approx \hat{A}$ via $\Phi_{\Lambda}$ where $\Phi_{\Lambda} \leq_{T} \Lambda \oplus \mathbf{0}^{(\mathbf{8})}$. 
Sketch. For $A_{T}$ the above construction gives us a $\mathbf{0}^{\prime \prime}$ (they are constructed on the true path) listing of the sets $D_{\chi}, R_{\chi, i}$, and $M_{\chi, i}$. So they are available for us to use here. The idea is to recover images of these sets on the hatted side. This recovery relies on Theorem 6.3 of Cholak and Harrington [8] that if $D$ and $\hat{D}$ are automorphic via $\Psi$, then $D$ and $\hat{D}$ are automorphic via $\Theta$ where $\Theta \uparrow \mathcal{E}(D)$ is $\Delta_{3}^{0}$, as well as more intricate material on extendible algebras (in particular, careful application of Theorem 5.10 of Cholak and Harrington [8]). Then using these recovered sets we constructed the desired automorphism as hinted to above. It takes $\Lambda \oplus \mathbf{0}^{(\mathbf{8})}$ to recover the needed sets. The construction of the automorphism needs the recovered sets and an oracle for $\mathbf{0}^{(2)}$.

\subsection{Properly $\Delta_{\alpha}^{0}$ orbits.}

Theorem 5.3 (Folklore $\left.^{6}\right)$. For all finite $\alpha$ there is a computable tree $T_{i_{\alpha}}$ from the list in Theorem 2.6 such that, for all computable trees $T, T$ and $T_{i_{\alpha}}$ are isomorphic iff $T$ and $T_{i_{\alpha}}$ are isomorphic via an isomorphism computable in $\operatorname{deg}(T) \oplus 0^{(\alpha)}$. But, for all $\beta<\alpha$ there is an $i_{\beta}^{*}$ such that $T_{i_{\beta}^{*}}$ and $T_{i_{\alpha}}$ are isomorphic but are not isomorphic via an isomorphism computable in $0^{(\beta)}$.

It is open if the above theorem holds for all $\alpha$ such that $\omega \geq \alpha<\omega_{1}^{\mathrm{CK}}$. But if it does then so does the theorem below.

Theorem 5.4. For all finite $\alpha>8$ there is a properly $\Delta_{\alpha}^{0}$ orbit.

Proof. Assume that $A_{T_{i_{\alpha}}}$ and $\hat{A}$ are automorphic via an automorphism $\Phi$. Hence, by part 2 of the game plan, $\mathcal{T}(\hat{A})$ and $T_{i_{\alpha}}$ are isomorphic. Since $\mathcal{T}(\hat{A})$ is computable in $0^{(8)}, \alpha>8$, and by Theorem $5.3, \mathcal{T}(\hat{A})$ and $T_{i_{\alpha}}$ via a $\Lambda \leq_{T} 0^{(\alpha)}$. By Theorem 5.2, $\hat{A}$ and $A_{T_{i_{\alpha}}}$ are automorphic via an automorphism computable in $0^{(\alpha)}$.

Fix $\beta$ such that $8 \geq \beta<\alpha$. By part 3 of the game plan and the above paragraph, $A_{T_{i_{\alpha}}}$ and $A_{T_{i_{\beta}^{*}}}$ are automorphic via an automorphism computable in $0^{(\alpha)}$. Now assume $A_{T_{i_{\beta}^{*}}} \approx A_{T_{i_{\alpha}}}$ via $\Phi$. By part 2 of the game plan, $\mathcal{T}\left(A_{T_{i_{\beta}^{*}}}\right) \cong T_{i_{\alpha}}$ via $\Lambda_{\Phi}$, where $\Lambda_{\Phi} \leq_{T} \Phi \oplus \mathbf{0}^{(\mathbf{2})}$. Since $\mathcal{T}\left(A_{T_{i_{\beta}^{*}}}\right)$ is computable in $0^{(8)}$ and $\mathcal{T}\left(A_{T_{i^{*}}}\right)$ is isomorphic to $T_{i_{\beta}^{*}}$ via an isomorphism computable in $0^{(\beta)}$ (part 1 of the game plan), by Theorem 5.3, $\Lambda_{\Phi}>_{T} 0^{(\beta)}$. Hence $\Phi>_{T} 0^{(\beta)}$.

\footnotetext{
${ }^{6}$ See Section 5 of the full paper [9] for more information and a proof.
} 
5.3. Our Orbits and Hemimaximal Degrees. Recall that set is hemimaximal iff it is the nontrivial split of a maximal set. A degree is hemimaximal iff it contains a hemimaximal set.

Let $T$ be given. Construction $A_{T}$ as above. For all $i$, either $A_{T}$ is hemimaximal in $R_{i}$ or $A_{T} \cap R_{i}$ is computable. If $A_{T}$ is hemimaximal in $R_{i}$ then $A_{T} \cap R_{i}$ is a split of maximal set $M \sqcup \bar{R}_{i}$ and hence $A_{T}=\left(A_{T} \cap R_{i}\right)$ is a hemimaximal set. $A_{T}=\bigsqcup_{i \in \omega}\left(A_{T} \cap R_{i}\right)$ where $A_{T} \cap R_{i}$ is either hemimaximal or computable. So the degree of $A_{T}$ is the infinite join of hemimaximal degrees. It is not known if the (infinite) join of hemimaximal degrees is hemimaximal. Moreover, this is not an effective infinite join. But if we control the degrees of $A_{T} \cap R_{i}$ we can control the degree of $A_{T}$. By modifying our proofs we can achieve the following degree controls.

Theorem 5.5. Let $H$ be hemimaximal. We can construct $A_{T}$ such that $A_{T} \equiv_{T} H$.

Indeed, we can show the following.

Theorem 5.6. There is an $A_{T}$ whose orbits contain a representative of every hemimaximal degree, and hence of all jump classes.

\section{REFERENCES}

[1] C. J. Ash and J. Knight. Computable structures and the hyperarithmetical hierarchy, volume 144 of Studies in Logic and the Foundations of Mathematics. North-Holland Publishing Co., Amsterdam, 2000. ISBN 0-444-50072-3. 2

[2] P. Cholak. The translation theorem. Arch. Math. Logic, 33:87-108, 1994. 4.1

[3] P. Cholak, R. Downey, and M. Stob. Automorphisms of the lattice of recursively enumerable sets: Promptly simple sets. Trans. Amer. Math. Soc., 332:555-570, 1992. 1

[4] Peter Cholak. The Computably Enumerable Sets: the Past, the Present and the Future. Theory and Applications of Models of Computation, 2006, Beijing China, Slides can be found at http://www.nd.edu/ cholak, 2006. 3

[5] Peter Cholak. Automorphisms of the lattice of recursively enumerable sets. PhD thesis, University of Wisconsin, 1991. 1

[6] Peter Cholak. Automorphisms of the lattice of recursively enumerable sets. Mem. Amer. Math. Soc., 113(541):viii+151, 1995. ISSN 0065-9266. 1 
[7] Peter Cholak and Leo Harrington. Isomorphisms of splits of computably enumerable sets. J. of Symbolic Logic, 68(3):1044-1064, 2003. 4

[8] Peter Cholak and Leo A. Harrington. Extension theorems, orbits, and automorphisms of the computably enumerable sets. To appear in Trans. Amer. Math. Soc. Final version as of 8/31/2005. math.LO/0408279. 2, 2, 2, 4, 4.1, 4.2, 4.1, 4.3, 4.1, 4.4, 4.5, 4.6, $4.2,4.2,5.1$

[9] Peter Cholak, Rod Downey, and Leo A. Harrington. On the Orbits of Computable Enumerable Sets. Submitted. math.LO/0607264. $2,2,4,4,6$

[10] Peter Cholak, Rod Downey, and Eberhard Herrmann. Some orbits for $\mathcal{E}$. Ann. Pure Appl. Logic, 107(1-3):193-226, 2001. ISSN 01680072. 4.2, 4.12

[11] Peter A. Cholak and Rod Downey. Invariance and noninvariance in the lattice of $\Pi_{1}^{0}$ classes. J. London Math. Soc. (2), 70(3):735-749, 2004. ISSN 0024-6107. 1

[12] Peter A. Cholak and Leo A. Harrington. Definable encodings in the computably enumerable sets. Bull. Symbolic Logic, 6(2):185196, 2000. A copy can be found at http://www.nd.edu/ cholak. 1

[13] Peter A. Cholak and Leo A. Harrington. On the definability of the double jump in the computably enumerable sets. J. Math. Log., 2 (2):261-296, 2002. ISSN 0219-0613. 4

[14] R. Downey and A. Montalbán. Slender classes. Submitted, 2006. 1

[15] R. G. Downey and M. Stob. Jumps of hemimaximal sets. Z. Math. Logik Grundlag. Math., 37:113-120, 1991. 3

[16] R. G. Downey and M. Stob. Automorphisms of the lattice of recursively enumerable sets: Orbits. Adv. in Math., 92:237-265, 1992. 1, 3, 3, 4.10

[17] Rod Downey and Leo Harrington. There is no fat orbit. Ann. Pure Appl. Logic, 80(3):277-289, 1996. ISSN 0168-0072. 1, 3

[18] R. M. Friedberg. A criterion for completeness of degrees of unsolvability. J. Symbolic Logic, 22:159-160, 1957. 1

[19] Sergey S. Goncharov, Valentina S. Harizanov, Julia F. Knight, and Richard A. Shore. $\Pi_{1}^{1}$ relations and paths through $\mathcal{O}$. J. Symbolic Logic, 69(2):585-611, 2004. ISSN 0022-4812. 3

[20] Leo Harrington and Robert I. Soare. Codable sets and orbits of computably enumerable sets. J. Symbolic Logic, 63(1):1-28, 1998. ISSN 0022-4812. 3 
[21] Leo A. Harrington and Robert I. Soare. Post's program and incomplete recursively enumerable sets. Proc. Nat. Acad. Sci. U.S.A., 88:10242-10246, 1991. 1, 1.3, 3

[22] Leo A. Harrington and Robert I. Soare. The $\Delta_{3}^{0}$-automorphism method and noninvariant classes of degrees. J. Amer. Math. Soc., 9(3):617-666, 1996. ISSN 0894-0347. 1, 3

[23] Leo A. Harrington and Robert I. Soare. Definability, automorphisms, and dynamic properties of computably enumerable sets. Bull. Symbolic Logic, 2(2):199-213, 1996. ISSN 1079-8986. 1

[24] Stephen C. Kleene and Emil L. Post. The upper semi-lattice of degrees of recursive unsolvability. Ann. of Math. (2), 59:379-407, 1954. 1

[25] Alistair H. Lachlan. On the lattice of recursively enumerable sets. Trans. Amer. Math. Soc., 130:1-37, 1968. 2.7, 2

[26] Manuel Lerman and Robert I. Soare. $d$-simple sets, small sets, and degree classes. Pacific J. Math., 87(1):135-155, 1980. ISSN 0030-8730. 1

[27] W. Maass. On the orbit of hyperhypersimple sets. J. Symbolic Logic, 49:51-62, 1984. 1, 2.12

[28] W. Maass and M. Stob. The intervals of the lattice of recursively enumerable sets determined by major subsets. Ann. Pure Appl. Logic, 24:189-212, 1983. 1

[29] S. S. Marchenkov. A class of incomplete sets. Math. Z., 20:473487, 1976. 2

[30] D. A. Martin. Classes of recursively enumerable sets and degrees of unsolvability. Z. Math. Logik Grundlag. Math., 12:295-310, 1966. 1

[31] A. A. Muchnik. On the unsolvability of the problem of reducibility in the theory of algorithms. Dokl. Akad. Nauk SSSR, N. S. 108: 194-197, 1956. 1

[32] Emil L. Post. Recursively enumerable sets of positive integers and their decision problems. Bull. Amer. Math. Soc., 50:284-316, 1944. 1

[33] Theodore A. Slaman and W. Hugh Woodin. Personal communication, 1989. 2.1

[34] Robert I. Soare. Automorphisms of the lattice of recursively enumerable sets I: maximal sets. Ann. of Math. (2), 100:80-120, 1974. $1,1,4.8,4.2,4.9$

[35] Robert I. Soare. Automorphisms of the lattice of recursively enumerable sets. Bull. Amer. Math. Soc., 80:53-58, 1974. 1

[36] Alan M. Turing. Systems of logic based on ordinals. Proc. London Math. Soc. (3), 45:161-228, 1939. 1 
[37] Rebecca Weber. A definable relation between c.e. sets and ideals. $\mathrm{PhD}$ thesis, University of Notre Dame, 2004. 1

[38] Rebecca Weber. Invariance in $\mathcal{E}^{*}$ and $\mathcal{E}_{\Pi}$. Trans. Amer. Math. Soc., 358(7):3023-3059 (electronic), 2006. ISSN 0002-9947. 1

[39] Walker M. White. Characterizations for Computable Structures. PhD thesis, Cornell University, Ithaca, NY, USA, 2000. 3

Department of Mathematics, University of Notre Dame, Notre DAME, IN 46556-5683

E-mail address: Peter.Cholak.1@nd.edu

URL: http://www.nd.edu/ cholak

School of Mathematics, Statistics and Computer Science, Victoria University, P.O. Box 600, Wellington, NeW Zealand

E-mail address: Rod.Downey@vuw.ac.nz

URL: http://www.mcs . vuw . ac.nz/ downey

Department of Mathematics, University of California, Berkeley, CA 94720-3840

E-mail address: leo@math.berkeley.edu 